\title{
Incidence and Predictors of Diabetes Mellitus And Outcome of Pre-Diabetes: A Prospective, Community-Based Cohort Follow-Up Study
}

Shamila Thivanshi De Silva ( $\square$ shamiladp@hotmail.com )

Faculty of Medicine, University of Kelaniya, Sri Lanka https://orcid.org/0000-0001-7241-0963

\section{Dileepa Ediriweera}

University of Kelaniya Faculty of Medicine

Madunil Anuk Niriella

University of Kelaniya Faculty of Medicine

Thulani Beddage

University of Kelaniya Faculty of Medicine

Anuradhani Kasturiratne

University of Kelaniya Faculty of Medicine

Arunasalam Pathmeswaran

University of Kelaniya Faculty of Medicine

\section{Norihiro Kato}

National Center for Global Health and Medicine: Kokuritsu Kenkyu Kaihatsu Hojin Kokuritsu Kokusai Iryo Kenkyu Center

Rajitha Wickramasinghe

University of Kelaniya Faculty of Medicine

H Janaka de Silva

University of Kelaniya Faculty of Medicine

\section{Research}

Keywords: Diabetes, Prediabetes, Incidence, Predictors, Outcome, Sri Lanka

Posted Date: September 2nd, 2020

DOI: https://doi.org/10.21203/rs.3.rs-69311/v1

License: (c) (1) This work is licensed under a Creative Commons Attribution 4.0 International License.

Read Full License 


\section{Abstract}

\section{Background}

There is limited data on prevalence and outcome of prediabetes (PDM) and incidence of type 2 diabetes mellitus (T2DM) from South Asia. We investigated this in an urban, adult population in Sri Lanka that was followed-up for seven years.

\section{Methods}

The study population (selected by age-stratified random sampling from the community) was initially screened in 2007 and re-evaluated in 2014. On both occasions they were assessed by structured interview, anthropometric measurements, liver ultrasound, biochemical and serological tests.

\section{Results}

In the original cohort of 2985 recruited in 2007 [54.8\% women, median age (IQR) 53 (47-59)], 737 had T2DM [baseline prevalence 24.7\% (95\% Cl: 23.1-26.2)] and 525 (17.7\%) had PDM [54.1\% women, median age (IQR) 56 (50-60)]. 2148/2985 (71.6\%) attended follow-up in 2014 [57.5\% women; median (IQR) 60 (54-66) years], which included 1650 who did not have T2DM in 2007. By 2014, 436/1650 (27.6\%) had developed new T2DM [annual incidence 3.9\% (95\% Cl:3.0-4.9). On logistic regression, PDM, central obesity, dyslipidemia and non-alcoholic fatty liver disease (NAFLD) showed significant association with incident T2DM. Of 525 with PDM in 2007, 365 (69.5\%) presented for follow up in 2014; 147 (40.3\%) remained in PDM, 201 (55.1\%) had progressed to T2DM and 17 (4.6\%) had reverted to normal. Annual conversion rate of PDM to T2DM was 7.9\%. Increase in waist circumference and decrease in HDL predicted progression to T2DM.

\section{Conclusions}

Presence of components of the metabolic syndrome at baseline predicted new-onset T2DM and conversion of PDM to T2DM. Targeted lifestyle interventions are essential for individuals with metabolic risk to prevent incident T2DM.

\section{Introduction}

Diabetes ranks seventh in the global burden of diseases ${ }^{1}$. The prevalence of type 2 diabetes mellitus (T2DM) among adults aged 20-79 years is expected to rise from 463 million in 2019 to 578 million by year 2030 and to 700 million by year $2045^{2}$. The highest percentage increases in disease prevalence are likely to be in non-Western and developing nations, with major increases in the Middle-East, Sub-Saharan Africa, India, Asia, and Latin America ${ }^{3}$. Asian countries currently contribute more than $60 \%$ of the world's diabetic population ${ }^{4}$, and India has been named the diabetes capital of the world 5 . 
Prediabetes (PDM) is an at-risk state for the development of T2DM ${ }^{6}$. It is defined as impaired fasting glucose (IFG) and/or impaired glucose tolerance (IGT) ${ }^{2}$. The American Diabetes Association defines PDM as HbA1c $5.7-6.4 \%^{7}$. People with either IFG or IGT have an increased risk of developing diabetes and a higher prevalence of cardiovascular disease than normoglycemic individuals ${ }^{8}$. PDM has an annual global conversion rate to T2DM of $5-10 \%^{6}$. However, information regarding the outcome of PDM in South Asia is scarce.

Obesity is a major predictor for the development of $\mathrm{T}^{2} \mathrm{DM}^{9}$. Obesity and T2DM are independent risk factors for the development of cardiovascular disease ${ }^{10}$, which is the leading cause of death worldwide among adults ${ }^{11}$. Early detection of modifiable risk factors (such as obesity), PDM and T2DM will help reduce disease burden in any community. Detecting at-risk populations and implementing risk reduction strategies is the mainstay for reducing incidence of diabetes worldwide ${ }^{12}$.

While there are many reports on the prevalence of T2DM, there are few on its incidence. There are not many community-based cohort studies reporting incident T2DM, and this is especially true of the South Asian region.

The Ragama Health Study (RHS) is a prospective study of non-communicable diseases carried out on a large community cohort. The RHS is a collaboration between the Faculty of Medicine, University of Kelaniya, Ragama, Sri Lanka and the National Centre for Global Health and Medicine, Tokyo, Japan ${ }^{13}$. The original cohort was recruited in 2007 and the study was conducted in the Ragama Medical Officer of Health $(\mathrm{MOH})$ area of the Gampaha district. The objective of the present study was to investigate the incidence and predictors of T2DM and the outcome of PDM in this cohort after seven years of follow-up.

\section{Methods}

The Ragama $\mathrm{MOH}$ administrative area is situated approximately $18 \mathrm{~km}$ north of Colombo, the commercial capital of Sri Lanka. Ragama is a bustling urban township with a multi-ethnic population. Participants of the RHS were resident adults, originally selected by age-stratified random sampling from electoral lists. The cohort was initially screened in 2007, when participants were aged 35-64 years, and was invited back for re-evaluation after 7 years, when participants were aged 42-71 years, in 2014.

In 2007 and 2014 all participants were assessed using a structured interview. They underwent clinical assessment and anthropometric measurements, ultrasound scanning of the liver was done and biochemical and serological tests were undertaken. Further details regarding the screening of the inception cohort are described elsewhere ${ }^{13}$. The follow up cohort was interviewed by trained personnel to obtain information on socio-demographic variables and lifestyle habits, including diet, alcohol consumption and physical activity. Medical records of subjects, whenever available, were analysed to obtain more details. Blood pressure (BP) and anthropometric parameters including height, weight and waist circumference (WC) were measured. Change in WC was classified as reduction $>5 \%$, between reduction $\leq 5 \%$ and increase $<5 \%$ (no change) and increase $\geq 5 \%$. Change in weight was classified as 
loss $>5 \%$, loss $\leq 5 \%$ and gain $<5 \%$ (no change), gain $\geq 5 \%$ and gain $\geq 10 \%$. Total body fat (TBF) and visceral fat percentage (VFP) were measured by a body composition monitor using bioelectrical impedance method (Omron HBF-362 body composition monitor, Omron Healthcare, Lake Forrest, Illinois, United States). Abnormal TBF was defined as $>32 \%$ for females and $>25 \%$ for males. Abnormal VFP was defined as $>10 \%$ for both genders ${ }^{14}$.

A10-mL sample of venous blood drawn from each participant was used to determine fasting serum triglycerides (TG), high density lipo-proteins (HDL), glycosylated hemoglobinA1c (HbA1c), hepatitis B surface antigen (HBsAg) and anti-hepatitis $\mathrm{C}$ virus antibodies (anti-HCV). Hepatitis serological tests were done using CTK Biotech ELISA kits. All participants also underwent ultrasonography of the liver with a 5$\mathrm{MHz} 50 \mathrm{~mm}$ convex probe (MindrayDP-10 Ultrasound Diagnostic Systems, Mindray Medical International Limited, Shenzhen, China). The ultrasound examination was carried out by medical personnel specially trained in liver ultrasonography. Non-alcoholic fatty liver disease (NAFLD) was diagnosed on established ultrasound criteria for fatty liver (two out of the following three criteria: increased echogenicity of the liver compared to kidney and spleen, obliteration of the vascular architecture of the liver and deep attenuation of the ultrasonic signal), safe alcohol consumption (Asian standards of $<14$ units/week for men and $<7$ units/week for females) and absence of hepatitis B and C markers ${ }^{15}$.

Data were entered in Epi Info 7 (Centres for Disease Control and Prevention, Atlanta, GA, USA) and logical and random checks were done. Statistical analysis was done using SPSS ver. 22.0 (SPSS, Chicago, IL, USA). Prevalence and incidence rates with $95 \%$ confidence intervals were calculated using standard formula for proportion estimates. Continuous and categorical data were described using median with interquartile range (IQR) and frequencies with percentages, respectively. Group comparisons were done using Mann Whitney U test and Pearson's Chi-square-test, for continuous and categorical variables, respectively.

Multiple linear logistic regression models were used to investigate the associated variables with incident DM. The following exposure variables in 2007 were investigated for association with incident DM: gender, age, educational level (i.e. less than General Certificate of Education Ordinary Level), income (i.e. less than median income in the cohort), unsafe alcohol use, smoking pack years, PDM, central obesity (waist circumference $\geq 90 \mathrm{~cm}$ for males and $\geq 80 \mathrm{~cm}$ for females), raised blood pressure (systolic BP $\geq 140$ or diastolic BP $\geq 90 \mathrm{mmHg}$, or treatment of previously diagnosed hypertension), low HDL [ $40 \mathrm{mg} / \mathrm{dL}$ $(1.03 \mathrm{mmol} / \mathrm{dL})$ in males and $<50 \mathrm{mg} / \mathrm{dL}(1.29 \mathrm{mmol} / \mathrm{dL})$ in females, or on specific treatment for this lipid abnormality], raised TG [ $\geq 150 \mathrm{mg} / \mathrm{dL}(1.7 \mathrm{mmol} / \mathrm{L})$ or on specific treatment for this lipid abnormality], dyslipidemia (total cholesterol $>140 \mathrm{mg} / \mathrm{dL}$, raised TG, raised HDL or on lipid-lowering therapy), NAFLD and percentage gain in body mass index (BMI), weight and waist circumference. Stepwise variable selection method was adopted to develop the final models. $\mathrm{P}<0.05$ was considered as significant.

All participants were enrolled after obtaining informed written consent. Ethical approval for the study was obtained from the Ethical Review Committee of the Faculty of Medicine, University of Kelaniya, Ragama, 
Sri Lanka.

\section{Results}

2985/3012 (99.1\%) [1636 women (54.8\%); median age (IQR) 53 (47-59) years] of the total participants recruited in 2007 had complete data for analysis. 2148/2985 (71.6\%) of the original cohort attended follow-up [1237 (57.5\%) women; median (IQR) 60 (54-66) years]. Those who attended follow up in 2014 were more likely to have central obesity and be female; other characteristics were similar in the inception and follow up cohorts (Table 1).

Table 1

Profile of the participants in 2007 who attended and did not attend follow up in 2014

\begin{tabular}{|c|c|c|c|}
\hline & $\begin{array}{l}\text { Attended follow-up } \\
\text { in } 2014 \\
(\mathrm{~N}=2148)\end{array}$ & $\begin{array}{l}\text { Did not attend } \\
\text { follow-up } 2014 \\
(\mathrm{~N}=837)\end{array}$ & $P$ value \\
\hline Males (\%) & $910(42.4)$ & 439 (52.4) & $<0.001$ \\
\hline Median age (IQR) & $53.0(47.0-59.0)$ & $53.0(46.0-59.7)$ & 0.630 \\
\hline Central obesity & 1213 (56.5\%) & 405 (48.4\%) & $<0.001$ \\
\hline Median FBS (IQR) & $104.0(96.0-117.0)$ & $104.5(97.0-124.0)$ & 0.035 \\
\hline Median SBP (IQR) & $132.0(119.0-147.0)$ & $132.0(120.0-147.0)$ & 0.461 \\
\hline Median DBP (IQR) & $78.0(71.0-87.0)$ & $79.0(72.0-87.9)$ & 0.340 \\
\hline Median TG (IQR) & $115.0(85.0-162.0)$ & $121.0(86.0-161.0)$ & 0.226 \\
\hline Median HDL (IQR) & $50.0(48.0-52.0)$ & $50.0(48.0-52.0)$ & 0.759 \\
\hline \multicolumn{4}{|c|}{$\begin{array}{l}\text { (IQR-inter quantile range, FBS-fasting blood sugar, SBP-systolic blood pressure, DBP-diastolic blood } \\
\text { pressure, TG-triglyceride, HDL-high density lipoprotein) }\end{array}$} \\
\hline \multicolumn{4}{|c|}{$\begin{array}{l}\text { Of the } 2985 \text { subjects in the inception cohort, } 737 \text { had established T2DM, giving a prevalence of } 24.7 \% \\
\text { ( } 95 \% \text { Cl: } 23.2 \%-26.3 \%) \text {. Those with established T2DM were significantly more likely to be older, have } \\
\text { a lower income, have a higher body weight, BMI and waist circumference, hypertension, coronary } \\
\text { artery disease, dyslipidemia and NAFLD, compared to those without T2DM (Table 2). }\end{array}$} \\
\hline \multicolumn{4}{|c|}{$\begin{array}{l}\text { 1650/2246 participants without T2DM in } 2007 \text { attended follow up in } 2014.436 / 1650(27.6 \%) \text { of } \\
\text { them had developed new-onset T2DM, giving an annual incidence of } 3.9 \%(95 \% \text { Cl: } 3.0 \%-4.9 \%) \text {. } \\
\text { Those with incident T2DM were less likely to be male, and more likely to have a higher body weight, } \\
\text { BMl and waist circumference, hypertension, dyslipidemia, NAFLD, coronary artery disease and pre- } \\
\text { diabetes in } 2007 \text {, compared to those who remained non-diabetic (Table } 3) \text {. }\end{array}$} \\
\hline
\end{tabular}


Table 2

- Characteristics of those with established diabetes, compared with those without diabetes in 2007

\begin{tabular}{|c|c|c|c|}
\hline \multirow[t]{4}{*}{ Parameter in 2007} & No Diabetes & Diabetes & \multirow[t]{4}{*}{$P$ value } \\
\hline & Number $(\%)$ or & Number (\%) or Median (IQR) & \\
\hline & Median (IQR) & $N=737$ & \\
\hline & \multicolumn{2}{|l|}{$N=2246$} & \\
\hline Age (years) & $52.0(46.0-58.0)$ & $55.0(50.0-60.0)$ & $<0.001$ \\
\hline Male gender & $1033(46.0)$ & 315 (42.7) & 0.134 \\
\hline Low education level & $1043(46.4)$ & $328(44.5)$ & 0.383 \\
\hline Household income $<$ median & $620(38.5)$ & $224(45.7)$ & 0.005 \\
\hline Smoking > 1 pack year & $333(14.8)$ & $85(11.5)$ & 0.030 \\
\hline Unsafe alcohol & $433(19.3)$ & $121(16.4)$ & 0.093 \\
\hline Weight (kg) & $58.4(51.0-66.5)$ & $60.8(53.8-68.3)$ & $<0.001$ \\
\hline Waist (cm) & $84.6(77.5-91.9)$ & $89.0(83.3-95.1)$ & $<0.001$ \\
\hline BMI $\left(\mathrm{kg} / \mathrm{m}^{2}\right)$ & $23.7(20.8-26.4)$ & $24.5(22.3-27.4)$ & $<0.001$ \\
\hline $\mathrm{BMI}>23 \mathrm{~kg} / \mathrm{m}^{2}$ & $1271(56.8)$ & $503(68.5)$ & $<0.001$ \\
\hline Central obesity & $1129(50.3)$ & $488(66.3)$ & $<0.001$ \\
\hline Hypertension & $908(40.0)$ & $434(58.8)$ & $<0.001$ \\
\hline Elevated TG or on treatment & 707 (98.5) & $417(97.9)$ & 0.623 \\
\hline Low HDL or on treatment & $594(26.8)$ & $270(37.5)$ & $<0.001$ \\
\hline Dyslipidemia & $1368(61.1)$ & $540(74.1)$ & $<0.001$ \\
\hline NAFLD & $614(37.8)$ & $325(74.5)$ & $<0.001$ \\
\hline Coronary artery disease & $71(3.2)$ & $63(8.5)$ & $<0.001$ \\
\hline
\end{tabular}


Table 3

-Comparison of characteristics between those with new-onset diabetes and those who remained nondiabetic in 2014

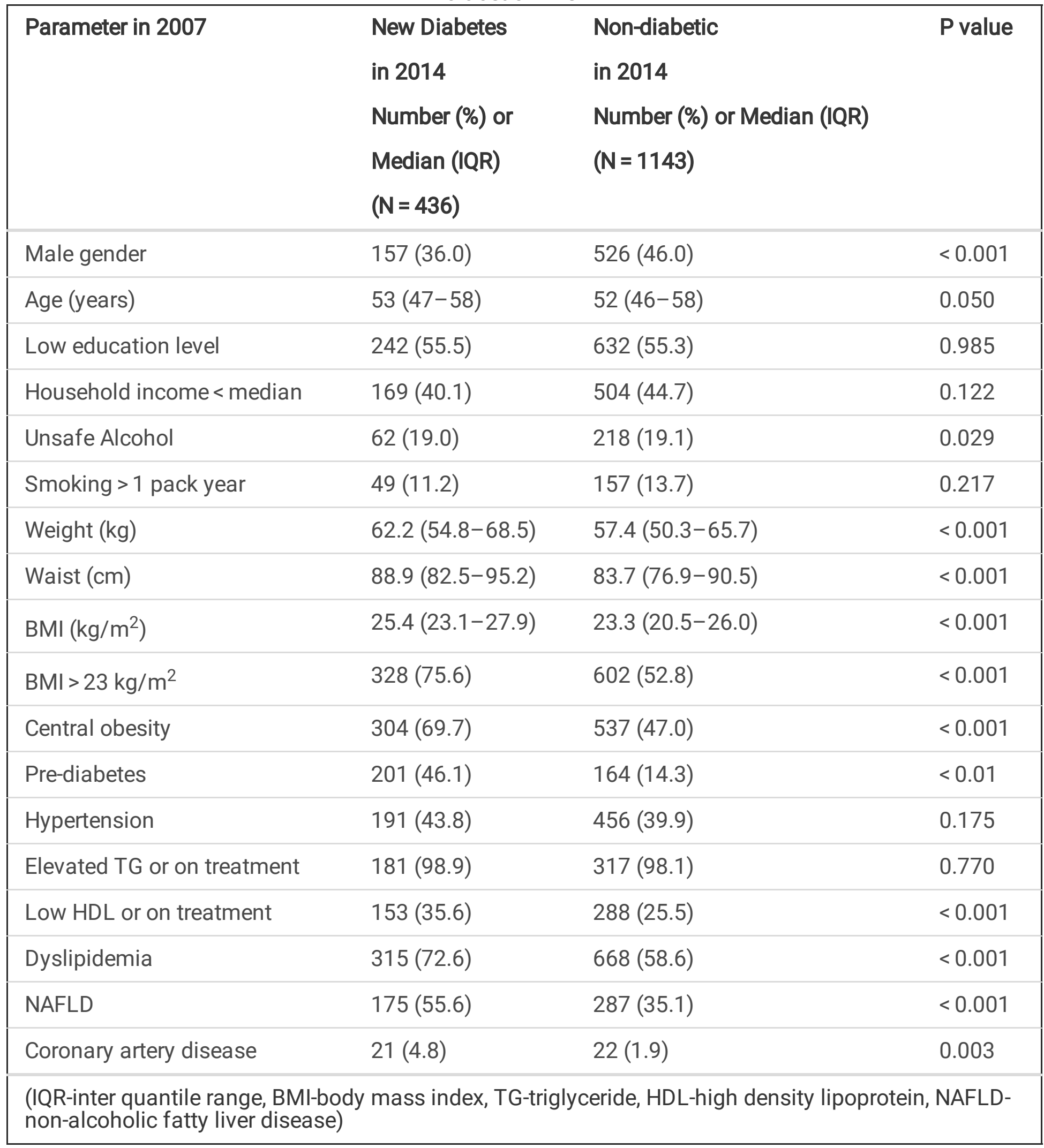

On logistic regression, pre-diabetes, central obesity, dyslipidemia and NAFLD showed significant association with incident T2DM (Table 4). 
Table 4

- Independent predictors of diabetes in those with no diabetes in 2007

\begin{tabular}{|llllll|}
\hline Variable - in 2007 & Coefficient & SE & p-value & OR & 95\% Cl for OR \\
\hline Central obesity & 0.560 & 0.169 & $<0.001$ & 1.751 & $1.258-2.438$ \\
\hline PDM & 1.491 & 0.154 & $<0.001$ & 4.441 & $3.286-6.003$ \\
\hline Dyslipidemia & 0.4311 & 0.1582 & 0.006 & 1.539 & $1.129-2.098$ \\
\hline NAFLD & 0.417 & 0.163 & 0.010 & 1.517 & $1.102-2.087$ \\
\hline $\begin{array}{l}\text { (PDM-prediabetes, NAFLD-non-alcoholic fatty liver disease, SE-standard error, OR-odds ratio, Cl- } \\
\text { confidence interval) }\end{array}$ & & & & \\
\hline
\end{tabular}

Abnormal VFP was observed among 234 (54.3\%) participants with new-onset T2DM and 426 (37.6\%) of non-T2DM participants in $2014(\mathrm{P}<0.001)$. Abnormal TBF was observed among $324(75.2 \%)$ new-onset T2DM participants and $801(70.6 \%)$ non-T2DM participants in $2014(\mathrm{P}=0.085)$.

Of the 525 participants with pre-diabetes in 2007, 365 (69.5\%) attended follow up in 2014. 201/365 (55.1\%) of them had developed T2DM, giving an annual conversion rate of pre-diabetes to T2DM of 7.9\%; $147(40.3 \%)$ remained in pre-diabetes and $17(4.6 \%)$ had reverted to normoglycemia. In those with prediabetes in 2007, increase in waist circumference [for each $1 \mathrm{~cm}$ increase OR $=1.04$ (95\% Cl: 1.02-1.07), $\mathrm{P}<0.001$ ] and decrease in $\mathrm{HDL}$ [for each unit decrease $\mathrm{OR}=1.91(95 \% \mathrm{Cl}: 1.13-3.26), \mathrm{P}=0.016$ ] predicted progression to diabetes.

\section{Discusson}

In this community cohort follow-up study of an urban, ageing adult population in Sri Lanka, the annual incidence of T2DM was 3.9\%. Pre-diabetes, central obesity, dyslipidemia and NAFLD showed association with new-onset T2DM. Abnormal VFP was also significantly more likely in those with new-onset T2DM. The annual conversion rate of pre-diabetes to T2DM was $7.9 \%$. Presence of components of the metabolic syndrome at baseline predicted conversion of PDM to T2DM and only a small proportion of PDM reverted to normoglycemia over seven years.

In China, the age-standardized incidence of T2DM was 9.5 per 1000 person-years for men and 9.2 for women from 2006 to $2014^{16}$. A retrospective nationwide longitudinal study in Taiwan during 1999 to 2004 showed that age-standardized prevalence of T2DM increased from 4.7 to $6.5 \%$ for men and from 5.3 to $6.6 \%$ for women ${ }^{17}$. In 2007 we reported a prevalence of $24.7 \%$ for T2DM in this adult population ${ }^{13}$; prevalence had increased to $52 \%$ after 7 years. The International Diabetes Federation (IDF) estimates that the age-adjusted comparative diabetes prevalence in adults aged $20-79$ years in Sri Lanka is $10.7 \%{ }^{2}$. The much higher prevalence in the present cohort highlights the increase in prevalence of T2DM with aging, which is a well-known phenomenon ${ }^{18}$. Despite may studies reporting on prevalence, to our 
knowledge, no community-based studies have previously investigated the incidence and predictors of T2DM in South Asian populations.

NAFLD, markers of obesity and impaired glucose tolerance are known to be positively associated with development of T2DM ${ }^{19}$. Several previous studies, including one from the present cohort, have shown that moderate to severe NAFLD is predictive of T2DM as it occurs in association with components of the metabolic syndrome, including insulin resistance ${ }^{20,21,22}$. Development of metabolic abnormalities and diabetes correlates with abdominal and visceral accumulation of adipose tissue, because visceral fat reduces insulin activity by increasing fatty acids. Visceral fat cells are known to release proteins that contribute to inflammation, atherosclerosis, dyslipidemia, and hypertension. It is likely that visceral adipose tissue is more closely associated with T2DM than other indices of obesity ${ }^{23}$. Several indices such as body mass index, waist circumference, waist-to-hip ratio, and waist-to-height ratio are used to determine general and central obesity, but waist-to-height ratio and waist circumference are better parameters in predicting diabetes as they directly correlate with the central obesity ${ }^{24}$. VFP as measured by bioelectrical impedance is an indirect measure of visceral fat deposition that corelates closely with the metabolic risk posed by obesity ${ }^{25}$. In our cohort, central obesity (as measured by WC), dyslipidemia and NAFLD at inception showed significant association with new-onset T2DM seven years later. Abnormal VFP was significantly more likely to be present in those with new-onset T2DM.

Prediabetes is a condition where blood glucose is above normal but does not measure up to the criteria to diagnose T2DM. Impaired glucose tolerance and impaired fasting glucose are collectively referred to as prediabetes. When patients have both IGT and IFG, their cumulative incidence of diabetes over 6 years is $65 \%$ more, compared to persons with normal blood glucose ${ }^{26,27}$. Identifying individuals with prediabetes together with targeted lifestyle interventions is an important and cost-effective method to prevent the future development of T2DM and its complications in a given population ${ }^{28,29}$. In our cohort the annual conversion rate of pre-diabetes to T2DM was $7.9 \%$, which is within the expected range of $5-10 \%$ for most populations ${ }^{6}$.

A fifth of those with PDM in the study cohort remained in pre-diabetes after seven years, while less than $5 \%$ reverted to normoglycemia, in the absence of a targeted, specific intervention. The Diabetes Prevention Program Outcomes Study (DPPOS) of 2014 demonstrated a 56\% reduction of the incidence of new-onset T2DM among individuals with prediabetes who reverted to normoglycemia with intensive lifestyle interventions ${ }^{30}$. Since increase in waist circumference and decrease in HDL predicted progression of PDM to T2DM in our cohort, interventions that target weight reduction are likely to be ideal for achieving regression of PDM to normoglycemia ${ }^{31}$.

There were several strengths in the design of this study. It was a relatively large, prospective, communitybased cohort follow-up study. The data collected prospectively at baseline in 2007 and at follow up in 2014 were robust and complete. More than 70\% of study participants attended follow-up after 7 years. There were, however, several limitations; TBF and VPF were not available at baseline for risk assessment, 
the follow-up sample consisted of mainly older adults, and although NAFLD was diagnosed based on accepted ultrasound criteria, inter-observer variability of the operators was not formally assessed.

Poorly controlled T2DM causes early mortality and morbidity through cardiovascular events, stroke, blindness, renal failure and lower limb amputation, that significantly impact quality of life. T2DM and its complications also impose a substantial economic burden on patients, their families, health systems and national economies through direct medical costs, loss of productivity and premature mortality ${ }^{32}$. Lifestyle interventions can effectively deter the progression of diabetes in individuals with impaired glucose tolerance.

\section{Conclusions}

This study demonstrates that the incidence and prevalence of T2DM in urban Sri Lankan adults are high and comparable to those reported from other parts of Asia. Presence of components of the metabolic syndrome predicted incident T2DM and conversion of prediabetes to T2DM, and only a small proportion of those with prediabetes reverted to normoglycemia. Our findings reiterate the need for targeted lifestyle interventions for individuals with metabolic risk, especially pre-diabetes, to prevent incident T2DM.

\section{Abbreviations}

anti-HCV - anti-hepatitis C virus antibodies

BMI - body mass index

BP - blood pressure

$\mathrm{Cl}$ - confidence interval

DBP - diastolic blood pressure

FBS - fasting blood sugar

HbA1c - glycosylated hemoglobin A1c

HBsAg - hepatitis B surface antigen

HDL - high density lipoprotein

IDF - International Diabetes Federation

IGT - impaired glucose tolerance

IFG - impaired fasting glucose

IQR - inter quantile range 
$\mathrm{MOH}$ - Medical Officer of Health

NAFLD - non-alcoholic fatty liver disease

$\mathrm{OR}$ - odds ratio

PDM - prediabetes

RHS - Ragama Health Study

SBP - systolic blood pressure

T2DM - type 2 diabetes mellitus

TBF - total body fat

TG - triglyceride

VFP - visceral fat percentage

WC - waist circumference

\section{Declarations}

Ethics approval and consent to participate - ethical approval was obtained from the Ethics Review Committee, Faculty of Medicine, University of Kelaniya. Informed written consent was obtained from all participants in 2007 and 2014.

Consent for publication - not applicable

Availability of data and material - The datasets used and analyzed during the current study are available from the corresponding author on reasonable request.

Competing interests - The authors declare that they have no competing interests.

Funding - We gratefully acknowledge grants from the Ministry of Higher Education of Sri Lanka and National Center for Global Health and Medicine, Tokyo, Japan for conduct of this study. The funding bodies played no role in the design of the study and collection, analysis, and interpretation of data and in writing the manuscript.

Author Contribution -SdeS and HJdeS conceptualized and designed the study. AK, SdeS, MAN, ARW, NK and HJdeS were involved in the establishment of the Ragama Health Study cohort. SdeS, MAN and AK collected data. DE analyzed the data assisted by TB, SdeS, AP, MAN and HJdeS. SdeS and HJdeS prepared and revised the manuscript. All authors read and agreed to the final version of the manuscript. 
Acknowledgments - We thank all those who have continuously supported the Ragama Health Study. We especially thank the participants of the study for their continued cooperation.

\section{References}

1. WHO Global Health Estimates. 2016: Deaths by Cause, Age, Sex, by Country and.

2. IDF Diabetes Atlas. 9th ed: International Diabetes Federation; 2019. www.diabetesatlas.org (accessed 8.8.2020).

3. WHO Diabetes Fact Sheet. World Health Organization;2018.

4. www.who.int/news-room/fact-sheets/detail/diabetes (accessed 8.8.2020).

5. Nanditha A, Ma RCW, Ramachandran A, Snehalatha C, Chan JCN, et al. Diabetes in Asia and the Pacific: Implications for the Global Epidemic. Diabetes Care. 2016;39(3):472-80.

6. Joshi SR, Parikh RM. India-diabetes capital of the world: now heading towards hypertension. J Assoc Physicians India. 2007;55:323-4.

7. Tubak AG, Herder C, Rathmann W, Brunner EJ, Kivimaki M. Prediabetes: a high risk state for diabetes development. Lancet Diabetes. 2012;379(9833):2279-90.

8. U.S. Department of Health and Human Services. National Institute of Diabetes and Digestive and Kidney Diseases. (November 2016). Risk Factors for Type 2 Diabetes. (accessed 8.8.2020).

9. Laakso M. Cardiovascular disease in type 2 diabetes from population to man to mechanisms: the Kelly West Award Lecture 2008. Diabetes Care. 2010;33(2):442-9.

10. Al-Goblan AS, Al-Alfi MA, Khan MZ. Mechanism linking diabetes mellitus and obesity. Diabetes Metab Syndr Obes. 2014;7:587-91.

11. Martín-Timón I, Sevillano-Collantes C, Segura-Galindo A, Del Cañizo-Gómez FJ. Type 2 diabetes and cardiovascular disease: Have all risk factors the same strength? World J Diabetes. 2014;5(4):44470.

12. World Health Organization Fact Sheet Cardiovascular Disease. 2017. www.who.int/news-room/factsheets/detail/cardiovascular-diseases-(cvds) (accessed 8.8.2020.

13. Bergman M, Buysschaert M, Schwarz PE, et al. Diabetes prevention: global health policy and perspectives from the ground. Diabetes Manag (Lond). 2012;2(4):309-21.

14. Dassanayake AS, Kasthuriratne A, Rindrajith S, et al. Prevalence and risk factors for non-alcoholic fatty liver disease among adults in an urban Sri Lankan population. J Gastroenterol Hepatol. 2009;24:1284-8.

15. De Silva ST, Niriella MA, Ediriweera DS, et al. Incidence and risk factors for metabolic syndrome among urban, adult Sri Lankans: a prospective, 7-year community cohort, follow-up study. Diabetol Metab Syndr. 2019;11:66.

16. Niriella MA, Pathmeswaran A, De Silva ST, et al. Incidence and risk factors for non-alcoholic fatty liver disease: A 7-year follow-up study among urban, adult Sri Lankans. Liver Int. 2017;37:1715-22. 
17. Wang $\mathrm{C}, \mathrm{Li} \mathrm{J}, \mathrm{Xue} \mathrm{H}$, et al. Type 2 diabetes mellitus incidence in Chinese: contributions of overweight and obesity. Diabetes Res Clin Pract. 2015;107(3):424-32.

18. Chang $\mathrm{CH}$, Shau WY, Jiang YD, et al. Type 2 diabetes prevalence and incidence among adults in Taiwan during 1999-2004: a national health insurance data set study.

19. Diabetes Medicine. 2010;27(6):636-43.

20. Kirkman MS, Briscoe VJ, Clark N, et al. Diabetes in older adults. Diabetes Care. 2012;35(12):265064.

21. Grønbaek H, Thomsen KL, Rungby J, et al. Role of nonalcoholic fatty liver disease in the development of insulin resistance and diabetes. Expert Rev Gastroenterol Hepatol. 2008;2:705-11.

22. Kotronen $\mathrm{A}$, Yki-Järvinen $\mathrm{H}$. Fatty liver: a novel component of the metabolic syndrome. Arterioscler Thromb Vasc Biol. 2008;28:27-38.

23. Kim CH, Park JY, Lee KU, et al. Fatty liver is an independent risk factor for the development of Type 2 diabetes in Korean adults. Diabet Med. 2008;25:476-781.

24. Kasturiratne A, Weerasinghe S, Dassanayake A, et al. Influence of non-alcoholic fatty liver disease on the development of diabetes mellitus. J of Gastroenterol Hepatol. 2013;28:142-7.

25. Fuster JJ, Ouchi N, Gokce N, et al. Obesity-Induced Changes in Adipose Tissue Microenvironment and Their Impact on Cardiovascular Disease. Circ Res. 2016;118(11):1786-807.

26. Lam BC, Koh GC, Chen C, et al. Comparison of Body Mass Index (BMI), Body Adiposity Index (BAI), Waist Circumference (WC), Waist-To-Hip Ratio (WHR) and Waist-To-Height Ratio (WHtR) as predictors of cardiovascular disease risk factors in an adult population in Singapore. PLoS One. 2015;10(4):e0122985.

27. Jung SH, Ha KH, Kim DJ. Visceral Fat Mass Has Stronger Associations with Diabetes and Prediabetes than Other Anthropometric Obesity Indicators among Korean Adults. Yonsei Med J. 2016;57(3):674-80.

28. Garber A, Handelsman Y, Einhorn D, et al. Diagnosis and management of prediabetes in the continuum of hyperglycemia - When do the risks of diabetes begin? A consensus statement from the American College of Endocrinology and the American Association of Clinical Endocrinologists. Endocr Pract. 2008;14:933-46.

29. American Diabetes Association. Standards of medical care in diabetes. Diabetes Care. 2015;38:10.

30. Herman WH. The economic costs of diabetes: Is it time for a new treatment paradigm? Diabetes Care. 2013;36:775-6.

31. Icks A, Haastert B, Gandjour A, et al. Cost-effectiveness analysis of different screening procedures for type 2 diabetes: The KORA Survey 2000. Diabetes Care. 2004;27:2120-8.

32. Perreault L, Pan Q, Mather KJ, et al. Effect of regression from prediabetes to normal glucose regulation on long-term reduction in diabetes risk: results from the Diabetes Prevention Program Outcomes Study. Lancet. 2012;379(9833):2243-51. 
33. Wing RR, Hamman RF, Bray GA, et al. Achieving weight and activity goals among diabetes prevention program lifestyle participants. Obes Res. 2004;12(9):1426-34. doi:10.1038/oby.2004.179.

34. WHO Global report on diabetes. World Health Organization. 2016. 